\title{
RELAÇÃO ENTRE CULTURA E CLIMA ORGANIZACIONAIS: UM ESTUDO EMPÍRICO EM UM CAMPUS DO INSTITUTO FEDERAL DE ENSINO
}

\author{
L. C. S. ROCHA ${ }^{1 *}$ e E. A. PELOGIO ${ }^{2}$ \\ ${ }^{1}$ Instituto Federal do Sul de Minas Gerais - IF Sul de Minas \\ ${ }^{2}$ Instituto Machadense de Ensino Superior - IMES \\ luiz.rocha@mch.ifsuldeminas.edu.br ${ }^{*}$
}

Artigo submetido em julho/2012 e aceito em outubro/2014

DOI: $10.15628 /$ holos.2014.977

\section{RESUMO}

O presente trabalho aborda o tema cultura e clima organizacionais em um Campus do Instituto Federal de Ensino situado em um estado do nordeste brasileiro, sendo produto de uma pesquisa que se constituiu a partir das questões: Como abordar a cultura e o clima organizacionais do Instituto Federal de Ensino? Até que ponto os fatores determinantes da cultura organizacional do Instituto Federal de Ensino influenciam os fatores determinantes de seu clima organizacional? Metodologicamente, trata-se de uma pesquisa quantitativa, com viés funcionalista, que utilizou-se de diversos testes estatísticos, tais como análise de clusters, análise discriminante, teste T-Student, análise de correlação de Pearson, análise de correlação de
Spearman e análise de correlação canônica. Buscou-se realizar uma amostra censitária, onde os questionários foram enviados a todos os servidores de um dos campus do Instituto Federal de Ensino sendo que obteve-se um retorno de $92 \%$ dos questionários enviados (57 dos 62 servidores participaram da pesquisa). A coleta de dados realizou-se por meio de questionário estruturado e autopreenchível enviado aos respondentes e devolvido aos pesquisadores pelo e-mail institucional. O texto conclui que houve forte influência da cultura organizacional sobre o clima organizacional, o que comprova empiricamente aquilo que é defendido por vários pesquisadores

PALAVRAS-CHAVE: Cultura Organizacional, Clima Organizacional, Organização Pública.

\section{RELATIONSHIP BETWEEN ORGANIZATIONAL CULTURE AND ORGANIZATIONAL CLIMATE: A STUDY IN A FEDERAL INSTITUTE OF EDUCATION'S CAMPUS}

\begin{abstract}
The present work approaches the theme organizational culture and organizational climate in a Federal Institute of Education's campus situated in a Brazilian northeast state, being product of a research that began in these questions: How does approach the organizational culture and the organizational climate in Federal Institute of Education? How does the Federal Institute of Education's organizational culture influence the organizational climate? Methodologically, this research is a quantitative research, with a functionalist bias, that used a lot of statistic tests, as clusters analysis, discriminant analysis, T-Student test, Pearson correlation analysis, Spearman
\end{abstract}

correlation analysis and canonic correlation analysis. This work looked for accomplishing a sample with all individuals in a Federal Institute of Education's campus and the surveys were answered by $92 \%$ of the public servants (57 of the 62 total public servants). The data collection was accomplished by structured and self-filled out survey sent to the answers and returned to the researchers by e-mail. The text concluded that there was a big influence of the organizational culture over the organizational climate, proving empirically what is defended by a lot of researchers

KEYWORDS: Organizational Culture, Organizational Climate, Public Organization. 


\section{INTRODUÇÃO}

A busca da sobrevivência organizacional tem mudado os paradigmas organizacionais, no sentindo de que, se antes era necessário motivar os funcionários, hoje se precisa buscar o seu comprometimento, o que faz com que as organizações despertem para a relevância da obtenção de ambientes positivos, onde os empregados e os grupos possam encontrar condições favoráveis para trabalharem mais eficazmente, aliando-se a isso a oportunidade de efetivos estímulos para o desenvolvimento pleno do indivíduo (MELLO, 2004). As atitudes dos funcionários e a capacidade destes de desenvolverem suas atividades em um nível de qualidade compatível com as exigências do mercado estão diretamente relacionadas à competitividade de uma organização. Assim, devese buscar os fatores que influenciam essas ações, sendo a cultura e o clima organizacionais tidos como alguns destes fatores (SOUZA, 1978, DEAL; KENNEDY, 1982, PETERS; WATERMAN JR., 1982, SANTOS, 1994, 1998, 2000, CAMERON; QUINN, 2006).

Ao se tratar de cultura e clima no ambiente organizacional, pensa-se na satisfação ou descontentamento dos seus membros, nos meios utilizados para atingir suas metas, isto é, buscase saber como está o clima da organização face à cultura desenvolvida ao longo do tempo. 0 trabalho desenvolvido nas organizações cria características próprias e se realiza com base nos princípios da cultura da organização (CASTRO, 2003).

Os conceitos e padrões culturais das organizações influenciam o surgimento do clima organizacional, que segundo Souza (1978), é considerado um conjunto de características que descrevem uma organização, um conglomerado de valores ou atitudes que afetam o relacionamento das pessoas ou, ainda, um fenômeno resultante dos elementos da cultura.

De maneira geral, os autores que estudam a cultura e o clima organizacionais por um viés funcionalista, consideram esses construtos como variáveis gerenciáveis e diretamente ligados ao desempenho das organizações, ou seja, são fatores mediadores de resultados organizacionais favoráveis. Sendo assim, o interesse pelo estudo da cultura e clima organizacionais encontra-se na tomada de consciência, por parte de teóricos e praticantes, da importância dos fatores culturais e do clima organizacional nas práticas de gestão e a crença no fato da cultura e do clima organizacionais constituírem fatores de diferenciação das organizações bem sucedidas.

Atualmente, as pesquisas que envolvem cultura e clima organizacionais têm se direcionado mais para as organizações voltadas ao mercado, mas também são sustentáveis para o universo das organizações públicas. Um estudo sobre cultura e clima organizacionais é bastante amplo e complexo e baseia-se em contribuições das Ciências Humanas ligadas principalmente, à Antropologia, Psicologia e Sociologia, apresentando, portanto, características multivariadas e interdisciplinares. Mas mesmo com estas e outras restrições, uma pesquisa de cultura e clima organizacionais pode ser útil e fundamental, pois oferece a possibilidade de um diagnóstico mais profundo e a partir daí, intervenções mais eficientes e eficazes, permitindo uma administração mais competente. Assim, este estudo tem como lócus um Campus do Instituto Federal de Ensino situado em um estado do nordeste brasileiro. É uma instituição centenária e possui um padrão em sua estrutura física e organizacional que pode ser identificado nacionalmente, quando comparado aos institutos de mesma natureza em outros Estados. 
O Instituto Federal de Ensino é uma organização com cultura própria, pois, possui estruturação formal definida, conjunto de regras, normas, práticas que são seguidas, distribuição do exercício do poder e controle entre seus membros, objetivos, metas, missões estabelecidas e que dirigem as ações, processos decisórios e comunicativos entre os membros desta comunidade organizacional, entre outras características. Esta instituição igualmente (re)produz e multiplica culturas organizacionais que podem influenciar seu clima organizacional. Esta situação gerou algumas indagações: Como abordar a cultura e o clima organizacionais num Instituto Federal de Ensino? Até que ponto os fatores determinantes da cultura organizacional do Instituto Federal de Ensino influenciam os fatores determinantes de seu clima organizacional? Diante destes questionamentos, o objetivo principal deste estudo é explorar a influência da cultura organizacional sobre o clima organizacional de um campus do Instituto Federal de Ensino situado em um estado do nordeste brasileiro.

Além dessa parte introdutória, este artigo está estruturado da seguinte forma: o capítulo 2 discorre sobre "Cultura Organizacional" e o 3 sobre "Clima Organizacional"; o capítulo 4 analisa as "Diferenças e Relações entre os Construtos Cultura e Clima Organizacionais"; o capítulo 5 aborda a "Estratégia Metodológica"; o capítulo 6 trata da "Análise e Discussão dos Resultados"; o capítulo 7 traz a "Conclusão" do estudo; e por fim apresentam-se as "Referências" utilizadas no trabalho.

\section{CULTURA ORGANIZACIONAL}

A literatura especializada está repleta de obras demonstrando particularidades marcantes entre diversas organizações existentes. Um dos aspectos mais importantes que provoca diferenças nestas instituições refere-se à cultura organizacional de cada uma delas. Assim, de certo modo, vários estudiosos deste tema concordam, em sua essência, que a cultura organizacional refere-se ao modo como as coisas são feitas em cada instituição, mas algumas definições são mais precisas e extensas que outras.

Para Cameron e Quinn (2006, p. 17) “cada cultura é, geralmente, refletida pela linguagem única, símbolos, regras e sentimentos etnocêntricos... Uma cultura da organização é refletida pelo que é valorizado, os estilos de liderança dominantes, a linguagem e os símbolos, os procedimentos e rotinas, e as definições de sucesso que fazem uma organização única".

Deal e Kennedy (1982) citam os valores, heróis, ritos, rituais e comunicações como elementos da cultura organizacional. Além disso, defendem que "uma cultura forte é um sistema de regras informais que indicam como as pessoas têm que se comportar na maior parte do tempo" (DEAL; KENNEDY, 1982, p. 15).

Peters e Waterman Jr. (1982) comentam que, no sentido organizacional, as "estórias, mitos ou lendas parecem ser muito importantes, pois transmitem os valores compartilhados pela organização, ou, a sua cultura" (PETERS; WATERMAN JR., 1982, p.75).

Hofstede (1991) define o termo "cultura organizacional", ao fazer analogia com o termo "cultura", como sendo a programação coletiva da mente que distingue os membros de uma organização dos de outra. Cita ainda que o conceito de "cultura de empresa é um conceito 'suave', intangível e holístico, com, no entanto, presumíveis consequências 'duras', tangíveis" (HOFSTEDE, 1991, p.34). O autor menciona, também, serem esses os "ativos psicológicos" de uma organização. 
Para Souza (1978, p.36) “cultura organizacional é o conjunto de fenômenos resultantes da ação humana, visualizada dentro das fronteiras de um sistema".

Santos $(1994,2000)$ define cultura organizacional como sendo os valores e crenças compartilhados pelos membros de uma organização, a qual funciona como um mecanismo de controle organizacional, informalmente aprovando ou proibindo comportamentos e que dá significado, direção e mobilização para os membros da organização.

Freitas (2005, p.97) entende a cultura organizacional:

primeiro como instrumento de poder; segundo, como conjunto de representações imaginárias sociais que se constroem e reconstroem nas relações cotidianas dentro da organização e que se expressam em termos de valores, normas, significados e interpretações, visando um sentido de direção e unidade, tornando a organização fonte de identidade e de reconhecimento para seus membros.

A autora ainda cita que um dos conceitos mais ricos na literatura sobre cultura organizacional (FREITAS, 1991) é o apresentado por Schein (1984, p.9):

Cultura organizacional é o conjunto de pressupostos básicos que um grupo inventou, descobriu ou desenvolveu ao aprender como lidar com seus problemas de adaptação externa e de integração interna, e que funcionam suficientemente bem para serem considerados válidos e ensinados a novos membros como a forma correta de perceber, pensar e sentir, em relação a esses problemas.

Diante do apresentado, percebe-se que existem inúmeras definições de cultura organizacional e que estas possuem alguns aspectos centrais que norteiam a discussão do tema. Além disso, por constituir-se em tema bastante subjetivo, tem-se o esforço dos acadêmicos em desenvolver diversos modelos, tipologias e identificar um conjunto de dimensões que em muito ajudam a organizar os atributos fundamentais do conceito. Entretanto, não há consenso quanto à metodologia de análise que mais se adéqua ao estudo de cultura organizacional, o que gera diferentes maneiras de analisar este construto. As duas principais vertentes epistemológicas de análise da cultura organizacional (SMIRCICH, 1983, CAMERON; QUINN, 2006) são: a funcionalista, que considera a cultura organizacional como algo que a organização possui (variável); e a interpretativista, que considera a cultura organizacional como algo que a organização é (metáfora). Assim, a vertente epistemológica funcionalista de análise da cultura organizacional, que foi adotada para o presente estudo, considera que a cultura é definida como um atributo possuído pelas organizações, podendo os pesquisadores e gestores identificar, mudar e medi-lo empiricamente, principalmente por métodos quantitativos. Além disso, esta perspectiva considera que a cultura organizacional é um potencial preditor de resultados organizacionais (tal como a eficácia), tendo como interesse subjacente a busca de previsibilidade, de controle organizacional.

Em consonância com esta vertente epistemológica foi escolhido o modelo denominado de Competing Value Model (CVM) para a análise da cultura, no presente estudo. O modelo assume que as organizações podem ser caracterizadas de acordo com os traços culturais ou dimensões comuns, sendo a identificação de aspectos que refletem valores e suposições centrais das organizações, a chave para avaliar a cultura organizacional (CAMERON; QUINN, 2006). Ao relacionar o enfoque organizacional, ao ambiente interno ou externo, e a estrutura organizacional, 
visando controle e estabilidade ou flexibilidade e mudança, emergem quatro tipos culturais: Cultura Clã ou Grupal; Cultura Hierárquica; Cultura de Adhocracia ou Inovativa; e Cultura de Mercado ou Racional. A representação gráfica das tipologias culturais são apresentadas na Figura 1.

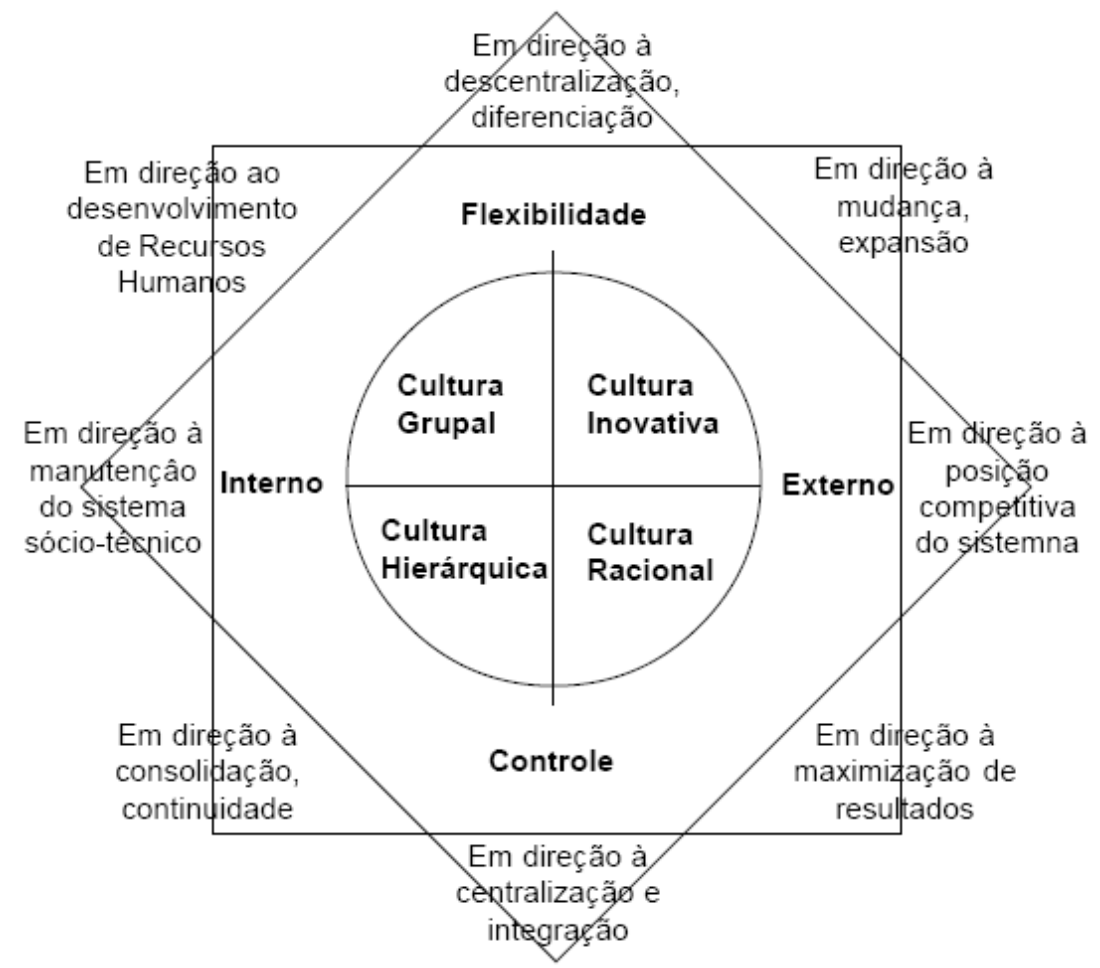

Figura 1 - Principais dimensões do Competing Value Model e os tipos de Cultura Organizacional Fonte: Santos, 1998.

A Cultura Clã ou Grupal enfatiza valores como coesão, moral, participação e abertura. As organizações que possuem este tipo de cultura costumam incentivar o trabalho em grupo, o programa de envolvimento dos colaboradores e o comprometimento corporativo com os empregados. Assim, os indivíduos apresentam-se altamente comprometidos com a organização e o desenvolvimento dos mesmos se traduz em benefícios a longo prazo (CAMERON; QUINN, 2006).

A Cultura Hierárquica possui como principal aspecto um local de trabalho formal e estruturado, com regras e políticas que mantêm a organização integrada e enfatizam a estabilidade, eficiência e previsibilidade. Existem padrões e procedimentos que determinam as atividades das pessoas e mantêm um ritmo estável de atividades. A liderança se dá através da coordenação e organização (CAMERON; QUINN, 2006).

A Cultura de Adhocracia ou Inovativa direciona para mudanças e novos desafios, acreditando que o sucesso está na produção de serviços e produtos únicos e originais. $O$ ambiente de trabalho é dinâmico, empreendedor e criativo. A liderança é visionária, inovativa e orientada para riscos. Ocorre a ênfase na atuação na fronteira do conhecimento, em produtos e/ou serviços e no crescimento rápido e aquisição de novos recursos. Ser o líder de um produto ou serviço é muito importante em culturas inovativas. A organização encoraja a liberdade e a iniciativa individual. O comprometimento com a experimentação e com a inovação é o que mantém a organização coesa (CAMERON; QUINN, 2006). 
Já na Cultura de Mercado ou Racional a organização é orientada para resultados. Ocorre a crença de que o ambiente externo não é benigno, e sim hostil, e que os consumidores realizam escolhas e estão interessados em valor. A principal tarefa da gerência é guiar a organização em direção à produtividade e ao alcance de metas e rendimentos. O principal negócio da organização é melhorar sua posição competitiva, assumindo que um propósito claro e uma estratégia agressiva levam a organização à produtividade e à rentabilidade (CAMERON; QUINN, 2006).

Cada uma dessas tipologias, tidas como ideais, é mensurada a partir de 6 variáveis, compondo assim um instrumento com 24 variáveis a serem respondidas utilizando-se uma escala Likert de 5 pontos. A média das 6 variáveis para cada tipologia cultural representa o quão presente a mesma está na organização. O CVM preconiza a coexistência das tipologias culturais nas organizações enfatizando que é de suma importância o equilíbrio entre culturas, pois, a ênfase em determinada cultura pode tornar a organização disfuncional (CAMERON; QUINN, 2006). O modelo também revela a importância da força cultural, que pode ser definida como a extensão em que os valores e crenças que permeiam a organização são ampla e intensamente compartilhados pelos seus membros (SANTOS, 1998), e estima que a cultura forte é aquela que mais incorpora as características de todos os quatro tipos culturais, possuindo médias elevadas em todas as tipologias a serem mensuradas.

\section{CLIMA ORGANIZACIONAL}

O termo clima organizacional, assim como outros termos relacionados à ciência administrativa, tem origem em outras disciplinas. Neste caso, o termo advém da meteorologia, configurando-se em uma "analogia meteorológica" (KATZ; KAHN, 1985, p.86).

Segundo Sá Leitão, Guimarães e Rosal (1998), existem na área do comportamento organizacional alguns assuntos que são de difícil compreensão e que se confundem em sua abrangência, sendo o clima organizacional um dos mais confusos e mal compreendidos. "A própria metáfora de onde se origina o termo clima denota a dificuldade em se precisar sua ocorrência e compreensão, quando se observa que as previsões do clima atmosférico dificilmente são precisas e seguras" (SÁ LEITÃO; GUIMARÃES; ROSAL, 1998, p.2).

O termo clima organizacional é bastante difuso, inclusive, quando se analisa seu surgimento como ramo de interesse da pesquisa em administração. Isto ocorre porque vários pesquisadores do comportamento humano nas organizações já tratavam do tema, mesmo que de maneira implícita desde os anos 30 do século XX.

O conceito é polêmico também por que muitos autores, a exemplo de Katz e Kahn (1985), o tratam como sinônimo de cultura, isto é, não existe consenso sobre definição de clima organizacional, apenas semelhanças de ideias, apesar do grande tempo decorrido desde o início das pesquisas nessa área. Oliveira (1995) argumenta que a expressão clima é relativamente nova, uma vez que nos primórdios da pesquisa sobre o tema usavam-se as expressões "atmosfera", "ambiente" ou "meio-ambiente" e isso pode ter colaborado para essa falta de consenso. No entendimento de Santos (1999, p.27):

O conceito de clima organizacional mostra um dilema em comum no esforço de descrever e compreender o comportamento humano. O problema é, em essência, o de operacionalizar o conceito para, então, medi-lo de forma confiável e válida. 
O que se evidencia, através dos trabalhos e pesquisas na área, é que existe um quase perfeito acordo entre os autores de que o clima organizacional é um conceito significativo, o qual tem importantes implicações para entender o comportamento humano nas organizações.

Assim, a comparação entre os conceitos de diversos autores se faz necessário para a identificação dos principais fatores envolvidos na análise do clima organizacional.

Forehand e Gilmer (1964, p. 362) definem clima organizacional como: “o conjunto de características que descrevem uma organização e que: a) distingue uma organização da outra; b) mantém-se de certa forma permanente e c) influencia o comportamento dos indivíduos na organização".

James e Jones (1974) propõem uma reconceituação do termo clima organizacional no sentido de diferenciar clima como um atributo individual e clima como um atributo organizacional. Ao se referir ao clima como um atributo individual, os autores defendem que deve ser empregada uma terminologia diferenciada, tal como "clima psicológico".

Souza (1978, p. 37), após apontar que a cultura organizacional é formada por três elementos componentes (preceitos, tecnologia e caráter) cita que:

Clima organizacional é um fenômeno resultante da interação dos elementos da cultura. É uma decorrência do peso de cada um dos elementos culturais e seu efeito sobre os outros dois. A excessiva importância dada à tecnologia, leva a um clima desumano; a pressão das normas cria tensão; a aceitação dos afetos, sem descuidar dos preceitos e o trabalho, leva a climas de tranquilidade e confiança, etc. E como cada um dos três elementos culturais é formado por diversos componentes, são inúmeras as combinações possíveis entre eles, criando-se climas de maior ou menor rigidez, realização ou emocionalidade.

Segundo Coda (1997), o clima organizacional reflete a tendência ou inclinação a respeito de até que ponto as necessidades da organização e das pessoas que dela fazem parte estão sendo efetivamente atendidas, sendo esse aspecto um dos indicadores da eficácia organizacional.

Com isso, Rizzatti (2002) defende que estudar clima organizacional é um excelente mecanismo para se conhecer a organização e o ambiente de trabalho, e assim transmitir aos dirigentes uma visão mais ampla da organização e não apenas uma simples interpretação de dados. Sob esse enfoque, o estudo do clima organizacional, seja por meio do estudo das percepções ou das aspirações dos indivíduos, é um diagnóstico adequado da instituição, especialmente pelo seu valor prático na adoção de novas estratégias administrativas. Ainda segundo este autor, para a realização de estudos sobre clima organizacional, o pesquisador dispõe de duas alternativas básicas: na primeira, ele adota um conjunto específico de fatores ou categorias já conhecidos e consagrados na literatura, ou elabora o seu próprio modelo.

Neste trabalho, será utilizada a Escala de Clima Organizacional (ECO) apresentada por Martins et al. (2004) e Martins (2008). A ECO é uma escala multidimensional construída e validada com o objetivo de avaliar a percepção do trabalhador sobre várias dimensões do clima organizacional. Esta escala é composta por 63 itens agrupados em cinco fatores denominados: apoio da chefia e da organização, recompensa, conforto físico, controle/pressão e coesão entre colegas. O fator "Apoio da chefia e da organização" refere-se ao suporte afetivo, estrutural e 
operacional da chefia e da organização fornecido aos empregados no desempenho diário de suas atividades no trabalho. Este fator é composto por 21 variáveis. O fator "Recompensa" sugere que diversas formas de recompensa são utilizadas pela empresa para premiar a qualidade, a produtividade, o esforço e o desempenho do trabalhador. Este fator é composto por 13 variáveis. O fator "Conforto físico" diz respeito ao ambiente físico, à segurança e ao conforto proporcionados pela empresa aos empregados, sendo composto por 13 variáveis. O fator "Controle/Pressão", composto por 9 variáveis, refere-se ao controle e pressão exercidos pela empresa e pelos supervisores sobre o comportamento e desempenho dos empregados. Por fim, o fator "Coesão entre colegas" exalta a união, vínculos e colaboração entre os colegas de trabalho, sendo formado por 7 variáveis. É importante destacar que este instrumento, assim como o utilizado para mensurar a cultura organizacional, utiliza uma escala de Likert de 5 pontos, os quais variam de "1-discordo totalmente" a "5-concordo totalmente".

\section{DIFERENÇAS E RELAÇÕES ENTRE OS CONSTRUTOS CULTURA E CLIMA ORGANIZACIONAIS}

Com o intuito de diferenciar os construtos cultura e clima organizacionais, recorre-se inicialmente a Puente-Palacios (2002). A autora argumenta que a semelhança entre os dois conceitos pode ser justificada pela compreensão de que são eles dois elementos de um mesmo cenário, a organização, mas não são o mesmo fenômeno: a cultura organizacional refere-se a um conjunto de normas, crenças, valores e mitos mais ou menos estáveis que define os padrões de comportamento esperado dos membros que a ela pertencem e são utilizados como modelos de comparação e julgamento, tendo sua importância pelo fato de nortear o comportamento e o desempenho dos seus membros; já o clima organizacional refere-se às representações compartilhadas das percepções dos membros sobre o agir da organização, construídas com base na cultura e de estabilidade menor, podendo mudar ao longo do tempo, mas, permanecendo o suficiente para serem significativas aos membros da organização, pois, não são características passageiras ou de estado de ânimo da organização, mas padrões de ação e reação. Entretanto, essa distinção nem sempre apareceu de forma clara, como já mencionado, em Katz e Kahn (1985).

Buscando a compreensão da diferença entre esses conceitos, apresenta-se a seguir, as categorias constitutivas de cada um, segundo Verbeke, Volgering e Hessels (1998). Esses autores analisaram a evolução conceitual das definições de clima organizacional (32 definições) e cultura organizacional (54 definições) a partir de 1964 e 1979, respectivamente. Cada definição foi analisada por uma comissão de peritos, cuja tarefa foi identificar as categorias constitutivas dos conceitos. Foram encontradas categorias estáveis para cada um, o que revelou a sua consistência por não aparecerem apenas de maneira esporádica, mas tratar-se de elementos constantes como se observa no Quadro 1.

As categorias comuns a ambos os conceitos demonstram que há espaço compartilhado entre eles, enquanto as excludentes revelam que não existe completa superposição entre ambos, o que leva a crer que ambos podem ser usados simultaneamente, sem medo de estar sendo redundante com relação aos fenômenos. Entretanto, Puente-Palacios (2002) questiona até que ponto essas categorias são suficientemente amplas para abarcar a totalidade do conceito e, ao mesmo tempo, suficientemente estreitas para diferenciá-lo de outros. A autora argumenta (PUENTE-PALACIOS, 2002, p.99) que "ainda que o clima seja composto por categorias conceituais 
comuns a outros eventos, a forma como elas se organizam e se relacionam constitui o elemento diferencial que estabelece a sua particularidade".

Quadro 1 - Categorias de cultura e clima organizacionais por ordem decrescente de frequência

\begin{tabular}{|c|l|c|l|}
\hline \multicolumn{2}{|c|}{ CLIMA ORGANIZACIONAL (32 definições) } & \multicolumn{2}{c|}{ CULTURA ORGANIZACIONAL (54 definições) } \\
\hline "Ranking" das Categorias & \multicolumn{1}{|c|}{ Categorias Conceituais } & "Ranking " das Categorias & \multicolumn{1}{|c|}{ Categorias Conceituais } \\
\hline 1 & ORGANIZAÇÃO & 1 & MEMBROS \\
\hline 2 & MEMBROS & 2 & COMPARTILHAR \\
\hline 3 & Percepções & 3 & Valores \\
\hline 4 & Características & 5 & ORGANIZAÇÃO \\
\hline 5 & COMPORTAMENTO & 6 & COMPORTAMENTO \\
\hline 6 & Descrições & 7 & Crenças \\
\hline 7 & COMPARTILHAR & 8 & Padrões \\
\hline 8 & CONJUNTO & 9 & Normas \\
\hline 9 & Influência & 10 & Aprender \\
\hline 10 & PRÁTICAS & 11 & Modo/Maneira \\
\hline & & 12 & Significados \\
\hline & & 13 & Sistema \\
\hline & & 14 & Suposições \\
\hline & & 15 & Social \\
\hline & & 16 & CONJUNTO \\
\hline & & 17 & PRÁTICAS \\
\hline & & & Compreensões \\
\hline
\end{tabular}

Fonte: adaptado de Verbeke, Volgering e Hessels (1998, p.314).

Nota: As palavras em letras maiúsculas representam as categorias comuns a ambos os conceitos.

Já para Cameron e Quinn (2006), o clima em comparação com a cultura se refere às atitudes, sentimentos e percepções mais temporárias por parte dos indivíduos. Cita, ainda, que a cultura organizacional é um atributo central e de lenta mudança das organizações, enquanto o clima, por se basear em atitudes, pode mudar mais rapidamente e dramaticamente. A cultura se refere o algo implícito, a aspectos frequentemente indiscerníveis das organizações, enquanto o clima se refere a atributos mais públicos, observáveis das organizações. A cultura inclui valores centrais e interpretações consensuais sobre como as coisas são, já o clima inclui perspectivas individualistas que são modificadas frequentemente de acordo com a mudança das situações e o surgimento de novas informações.

Ainda nesta discussão, Souza (1978) defende que o clima é uma resultante das variáveis culturais. Quando estas são alteradas, ocasionam alterações no clima. Curiosamente, o clima é mais perceptível do que suas fontes causais. É comparável a um perfume: percebe-se o efeito, sem conhecer os ingredientes, embora, às vezes seja possível identificar alguns deles. Outros autores como Schneider (1985), Tamayo (1999) e Martins (et al. 2004, 2008) compartilham dessa mesma ideia, afirmando ser o clima organizacional mais superficial do que a cultura e diretamente ligada a esta.

Assim, percebe-se que o conceito de clima difere do de cultura organizacional, embora, ambos estejam relacionados. Além disso, baseado no exposto, tem-se que o clima organizacional é a manifestação ou expressão da própria cultura (SOUZA, 1978, MARTINS et al., 2004, MARTINS, 2008). 


\section{ESTRATÉGIA METODOLÓGICA}

O método utilizado para a elaboração desse estudo foi o quantitativo, principalmente por uma exigência epistemológica do estudo de cultura e clima organizacionais, quando se utiliza um viés funcionalista. Este método, que leva a se caracterizar o estudo como sendo conclusivo-causal (MALHOTRA, 2001), tem a visão estrutural mais precisa e rígida, além de permitir a análise estatística e o estabelecimento de relações de causa e efeito entre variáveis. O presente estudo ainda pode ser caracterizado como um estudo de caso, com relação aos seus meios (VERGARA, 2000).

Buscou-se realizar uma amostra censitária, onde os questionários foram enviados a todos os servidores de um campus do Instituto Federal de Ensino sendo que obteve-se um retorno de 92\% dos questionários enviados (57 dos 62 servidores participaram da pesquisa). O questionário foi enviado aos respondentes e devolvido aos pesquisadores pelo e-mail institucional no período de fevereiro a março de 2010.

Com relação à coleta de dados, esta realizou-se por meio de questionário estruturado e auto-preenchível, através do qual foram coletados os dados sobre a cultura e o clima organizacionais do Instituto Federal de Ensino. Com o intuito de promover análises quantitativas a partir dos dados coletados, utilizou-se, como já mencionado, uma escala do tipo Likert de 5 pontos, sendo que o valor 1 correspondia a "discordo totalmente" e o 5 a "concordo totalmente". Neste estudo, não haverá criação de novas categorias e sim aplicação de categorias anteriormente desenvolvidas por outros autores.

A estruturação e montagem final do questionário dividiu-se em três partes. A primeira delas consistiu em informações que abrangiam aspectos relacionados aos respondentes como: sexo, idade, estado civil, anos de instituição, cargo ocupado, se exercia função de chefia e escolaridade. A segunda constou de um conjunto de 24 proposições que compõem o CVM. A terceira e última parte do questionário apresentava as 63 variáveis de mensuração do clima organizacional que compõem a ECO.

Para a análise dos dados foram utilizados dois programas estatísticos, o SPSS $^{\oplus}$ e o STATISTICA ${ }^{\circledR}$, sendo consideradas as seguintes etapas:

- Determinação das estatísticas descritivas das variáveis pessoais dos respondentes;

- Análise de Clusters, com base nas avaliações acerca da cultura organizacional;

- Determinação da média e desvio-padrão dos valores de cultura e clima organizacionais para os diferentes grupos definidos pela Análise de Clusters, com posterior teste estatístico (Teste T-Student) para evidenciar diferença estatisticamente significante entre as médias destes grupos;

- Análise discriminante dos grupos definidos pela Análise de Cluster, utilizando os valores da cultura organizacional, para verificar se estes foram corretamentes divididos; 
- Análise de correlação linear (Pearson e Spearman) entre os valores de cultura e clima organizacionais e os dados de caracterização dos respondentes, e teste T-Student para as variáveis sexo e "ocupa, ou não, função de chefia";

- Análise de correlação canônica, entre os fatores determinantes da cultura e do clima organizacional, visando verificar como estes se relacionam. Os fatores de clima foram considerados como variáveis dependentes e os fatores de cultura foram considerados como variáveis independentes. Esta técnica se apresenta como "a técnica mais adequada e poderosa" (HAIR JR. et al., 2007, p.362) quando se tem múltiplas variáveis dependentes e independentes.

\section{ANÁLISE E DISCUSSÃO DOS RESULTADOS}

Ao se analisar os 57 questionários respondidos pelos servidores do Instituto Federal de Ensino, obteve-se os seguintes valores para as estatísticas descritivas das variáveis de identificação dos respondentes: $61,4 \%$ homens e 38,6\% mulheres; $10,5 \%$ com idade entre 18 e 24 anos, 45,6\% com idade entre 25 e 34 anos, $22,8 \%$ com idade entre 35 e 44 anos e $21,1 \%$ com 45 anos ou mais; $47,4 \%$ solteiros, 49,1\% casados e 3,5\% divorciados; $19,3 \%$ com menos de 1 ano de instituição, $38,6 \%$ com 1 até 3 anos de instituição, 29,8\% com 3 até 20 anos de instituição e 12,3\% com 20 até 40 anos de instituição; $28,1 \%$ ocupavam cargo de assistentes administrativo, 8,8\% de técnicos de nível médio, $17,5 \%$ de técnicos de nível superior e $45,6 \%$ de docentes; $28,1 \%$ exerciam função de chefia e $71,9 \%$ não; e $12,3 \%$ possuíam o ensino médio, 3,5\% curso técnico, 33,3\% graduação, 12,3\% especialização, 31,6\% mestrado e 7,0\% doutorado.

Com a intenção de evidenciar a força cultural e os resultados dos fatores de clima organizacional para a organização estudada foram elaboradas as tabelas 1 e 2, com base nas respostas dos 57 servidores que participaram da pesquisa. A força cultural foi calculada a partir do somatório das médias para cada tipologia cultural. Já o valor para o clima é uma média de todos os seus fatores.

Tabela 1 - Resultados para a Cultura Organizacional

\begin{tabular}{|l|c|c|}
\hline \multicolumn{1}{|c|}{ ITENS } & MÉDIA & DESVIO PADRÃO \\
\hline Cultura Clã & 3,2281 & 0,6818 \\
\hline Cultura Inovativa & 3,3363 & 0,6626 \\
\hline Cultura de Mercado & 3,1959 & 0,6615 \\
\hline Cultura Hierárquica & 3,0175 & 0,5370 \\
\hline Força Cultural & 12,7778 & 1,9149 \\
\hline
\end{tabular}

Fonte: Dados da pesquisa, 2010.

Tabela 2 - Resultados para o Clima Organizacional

\begin{tabular}{|l|c|c|}
\hline \multicolumn{1}{|c|}{ ITENS } & MÉDIA & DESVIO PADRÃO \\
\hline Apoio da chefia & 3,4536 & 0,6593 \\
\hline Recompensa & 2,6073 & 0,6247 \\
\hline Conforto físico & 3,4494 & 0,5763 \\
\hline Controle/Pressão & 2,6374 & 0,6582 \\
\hline Coesão entre colegas & 3,6241 & 0,5654 \\
\hline Média Clima & 3,1544 & 0,4283 \\
\hline
\end{tabular}

Fonte: Dados da pesquisa, 2010. 
A partir desta primeira análise foi então realizada a análise de Clusters com o intuito de reunir os respondentes com perfis culturais semelhantes em grupos considerados distintos. A Tabela 3 mostra o resultado da análise de Clusters, apresentando a média e o desvio-padrão, tanto para a força cultural como para o clima, dos dois grupos formados.

Tabela 3 - Caracterização dos Grupos formados pela Análise de Clusters

\begin{tabular}{|c|c|c|c|c|c|}
\hline \multirow{2}{*}{ GRUPOS } & \multirow{2}{*}{ INDIVÍDUOS } & \multicolumn{2}{|c|}{ MÉDIA } & \multicolumn{2}{c|}{ DESVIO PADRÃO } \\
\cline { 3 - 6 } & & CULTURA & CLIMA & CULTURA & CLIMA \\
\hline 1 & 30 & 11,400 & 2,955 & 1,276 & 0,439 \\
\hline 2 & 27 & 14,309 & 3,376 & 1,208 & 0,301 \\
\hline
\end{tabular}

Fonte: Dados da pesquisa, 2010.

Com base na Tabela 3, nota-se que o primeiro grupo, com 30 indivíduos, apresenta força cultural menor que o segundo grupo, sendo por isso denominado grupo de cultura "fraca". Consequentemente, o segundo grupo, com 27 indivíduos, foi denominado grupo de cultura "forte". Para verificar se a cultura realmente discriminava os dois grupos, realizou-se a análise discriminante, que obteve $100 \%$ de acerto, comprovando o sucesso da análise de Clusters. Ao se analisar, na mesma tabela, os valores do clima organizacional para os dois grupos, evidencia-se que o grupo de cultura "forte" apresenta valores substancialmente maiores. Entretanto, as diferenças, tanto para cultura quanto para clima, não são suficientes para garantir sua representatividade. Para isto, o teste T-Student foi realizado, tanto para os valores da cultura quanto para os valores de clima, testando a hipótese nula de que a média das duas populações são iguais. Os resultados são apresentados na Tabela 4.

Tabela 4 - Teste T-Student

\begin{tabular}{|c|c|c|c|c|c|c|c|c|}
\hline \multirow{2}{*}{ GRUPOS } & \multicolumn{2}{|c|}{ MÉDIA } & \multicolumn{2}{c|}{ ERRO PADRÃO } & \multicolumn{2}{c|}{ ESTATÍSTICA T } & \multicolumn{2}{c|}{ SIG. } \\
\cline { 2 - 9 } & CULTURA & CLIMA & CULTURA & CLIMA & CULTURA & CLIMA & CULTURA & CLIMA \\
\hline 1 & 11,400 & 2,955 & 0,233 & 0,080 & \multirow{2}{*}{$-8,813$} & $-4,181$ & \multirow{2}{*}{0,000} & 0,000 \\
\hline 2 & 14,309 & 3,376 & 0,232 & 0,058 & & \\
\hline
\end{tabular}

Fonte: Dados da pesquisa, 2010.

Com base nos resultados do Teste T-Student rejeita-se a hipótese nula de que as médias dos grupos 1 e 2 para os valores de cultura e clima são iguais (sig.=0,000). Este resultado permite afirmar que as diferenças entre os valores para forças culturais e para o clima são estatisticamente significantes e que o grupo de cultura "forte" possui valores maiores para o clima organizacional, evidenciando que o grupo de cultura "forte" tem uma melhor percepção sobre o clima da organização. Este resultado empírico reforça o defendido por vários outros autores, como PuentePalacios (2002), Santos (1999), Souza (1978), Cameron e Quinn (2006), Martins et al. (2004) e Martins (2008). De uma forma geral estes autores defendem que a cultura influencia diretamente o clima organizacional, sendo o clima expressão da própria cultura.

Esta evidência da influência da cultura sobre o clima organizacional da instituição pesquisada ainda encontra respaldo no próprio modelo cultural, o CVM, uma vez que este defende que uma cultura forte está associada com a homogeneidade de esforços, clareza de foco e unicidade organizacional (CAMERON; QUINN, 2006), o que pode promover um ambiente amistoso dentro da organização.

Porém, suscita discussões sobre influências de outros fatores sobre esta relação, como no caso do estudo desenvolvido por Santos (1999) onde fatores como sexo, estado civil e tempo de trabalho na organização influenciaram a percepção dos indivíduos com relação ao clima. Para 
avaliar a influência dos fatores pessoais na percepção dos indivíduos acerca da cultura e do clima organizacionais na instituição pesquisada, foram realizados três testes estatísticos: correlação de Pearson entre os valores de cultura organizacional, clima organizacional, idade dos respondentes e tempo de trabalho na instituição; correlação de Spearman entre os valores de cultura organizacional, clima organizacional, estado civil, cargo e escolaridade; e o teste T-Student entre os valores auferidos para a cultura e clima organizacionais por grupos distintos, separados por sexo (masculino e feminino) e por ocupar, ou não, função de chefia. Os resultados estão dispostos nas tabelas 5,6 e 7 .

Tabela 5 - Correlação de Pearson

\begin{tabular}{|c|c|c|c|c|c|}
\hline & & CULTURA & CLIMA & IDADE & $\begin{array}{c}\text { TEMPO } \\
\text { DE } \\
\text { INSTITUIÇÃO }\end{array}$ \\
\hline \multirow{3}{*}{ CULTURA } & Coeficiente de Correlação & 1,000 & $0,684 * *$ & 0,010 & 0,044 \\
\hline & Significância & , & 0,000 & 0,940 & 0,747 \\
\hline & $\mathrm{N}$ & 57 & 57 & 57 & 57 \\
\hline \multirow{3}{*}{ CLIMA } & Coeficiente de Correlação & $0,684 * *$ & 1,000 & $-0,088$ & 0,030 \\
\hline & Significância & 0,000 & , & 0,517 & 0,826 \\
\hline & $\mathrm{N}$ & 57 & 57 & 57 & 57 \\
\hline \multirow{3}{*}{ IDADE } & Coeficiente de Correlação & 0,010 & $-0,088$ & 1,000 & $0,820 * *$ \\
\hline & Significância & 0,940 & 0,517 & , & 0,000 \\
\hline & $\mathrm{N}$ & 57 & 57 & 57 & 57 \\
\hline TEMPO & Coeficiente de Correlação & 0,044 & 0,030 & $0,820 * *$ & 1,000 \\
\hline $\mathrm{DE}$ & Significância & 0,747 & 0,826 & 0,000 & \\
\hline INSTITUIÇÃO & $\mathrm{N}$ & 57 & 57 & 57 & 57 \\
\hline
\end{tabular}

** Correlação significante ao nível de $1 \%$.

Fonte: Dados da pesquisa, 2010.

Tabela 6 - Correlação de Spearman

\begin{tabular}{|c|c|c|c|c|c|c|}
\hline & & CULTURA & CLIMA & $\begin{array}{c}\text { ESTADO } \\
\text { CIVIL }\end{array}$ & CARGO & ESCOLARIDADE \\
\hline \multirow{3}{*}{ CULTURA } & Coeficiente de Correlação & 1,000 & $0,621 * *$ & $-0,182$ & $-0,143$ & $-0,232$ \\
\hline & Significância & & 0,000 & 0,175 & 0,288 & 0,082 \\
\hline & $\mathrm{N}$ & 57 & 57 & 57 & 57 & 57 \\
\hline \multirow{3}{*}{ CLIMA } & Coeficiente de Correlação & $0,621 * *$ & 1,000 & $-0,176$ & $-0,158$ & $-0,215$ \\
\hline & Significância & 0,000 & & 0,191 & 0,240 & 0,109 \\
\hline & $\mathrm{N}$ & 57 & 57 & 57 & 57 & 57 \\
\hline \multirow{3}{*}{ ESTADO CIVIL } & Coeficiente de Correlação & $-0,182$ & $-0,176$ & 1,000 & $0,481 * *$ & $0,455 * *$ \\
\hline & Significância & 0,175 & 0,191 & & 0,000 & 0,000 \\
\hline & $\mathrm{N}$ & 57 & 57 & 57 & 57 & 57 \\
\hline \multirow{3}{*}{ CARGO } & Coeficiente de Correlação & $-0,143$ & $-0,158$ & $0,481 * *$ & 1,000 & $0,845 * *$ \\
\hline & Significância & 0,288 & 0,240 & 0,000 & & 0,000 \\
\hline & $\mathrm{N}$ & 57 & 57 & 57 & 57 & 57 \\
\hline \multirow{3}{*}{ ESCOLARIDADE } & Coeficiente de Correlação & $-0,232$ & $-0,215$ & $0,455 * *$ & $0,845 * *$ & 1,000 \\
\hline & Significância & 0,082 & 0,109 & 0,000 & 0,000 & \\
\hline & $\mathrm{N}$ & 57 & 57 & 57 & 57 & 57 \\
\hline
\end{tabular}

Correlação significante ao nível de $1 \%$.

Fonte: Dados da pesquisa, 2010. 
Tabela 7 - Teste T-Student

\begin{tabular}{|c|c|c|c|c|c|c|}
\hline \multirow{2}{*}{$\begin{array}{c}\text { FUNÇÃO DE } \\
\text { CHEFIA }\end{array}$} & \multicolumn{2}{|c|}{ MÉDIA } & \multicolumn{2}{|c|}{ ESTATÍSTICA F } & \multicolumn{2}{|c|}{ SIG. } \\
\hline & CULTURA & CLIMA & CULTURA & CLIMA & CULTURA & CLIMA \\
\hline Sim & 13,385 & 3,294 & \multirow{2}{*}{2,291} & \multirow{2}{*}{2,384} & \multirow{2}{*}{0,136} & \multirow{2}{*}{0,128} \\
\hline Não & 12,541 & 3,100 & & & & \\
\hline \multirow{2}{*}{ SEXO } & \multicolumn{2}{|c|}{ MÉDIA } & \multicolumn{2}{|c|}{ ESTATÍSTICA F } & \multicolumn{2}{|c|}{ SIG. } \\
\hline & CULTURA & CLIMA & CULTURA & CLIMA & CULTURA & CLIMA \\
\hline Masculino & 13,005 & 3,128 & \multirow{2}{*}{1,280} & \multirow{2}{*}{0,337} & \multirow{2}{*}{0,263} & \multirow{2}{*}{0,564} \\
\hline Feminino & 12,417 & 3,197 & & & & \\
\hline
\end{tabular}

Fonte: Dados da pesquisa, 2010.

Os resultados para as correlações de Pearson e Spearman indicam forte correlação positiva e estatisticamente significante entre a cultura e o clima organizacionais. Entretanto, quando analisa-se a relação dos valores atribuídos à cultura e ao clima organizacionais com outros fatores como idade, tempo de trabalho na instituição, estado civil, cargo e escolaridade, não há correlação estatisticamente significante (sig.>0,01), o que leva a crer na ausência de relação entre os mesmos. Pode-se inferir, também, que fatores como o sexo e o fato de ocupar, ou não, funções de chefia não influenciam nos valores atribuídos pelos respondentes à cultura e clima organizacionais, uma vez que as diferenças das médias para os diferentes grupos não foram estatisticamente significantes (ver Tabela 7). Esses resultados vão de encontro aos resultados obtidos por Santos (1999). Naquele caso era estudado somente o clima e a autora comprovou a influência de vários desses fatores nas repostas atribuídas pelos entrevistados.

Por último, fez-se a análise de correlação canônica entre os fatores de cultura e clima organizacionais. Utilizando-se dos indícios empíricos de que uma cultura "forte" influencia positivamente o clima organizacional, procurou-se saber a relação entre os fatores constituintes de cada construto. Fez-se a análise considerando os fatores de cultura organizacional como variáveis independentes $\left(X_{i}\right)$ e os fatores de clima organizacional como variáveis dependentes $\left(Y_{i}\right)$. Assim, o conjunto preditor ou explicativo será formado por "Cultura Clã", "Cultura Inovativa", "Cultura de Mercado" e "Cultura Hierárquica", enquanto o conjunto critério ou explicado será formado por "Apoio da chefia e da organização", "Recompensa", "Conforto físico", "Controle/Pressão" e "Coesão entre colegas". A Tabela 8 apresenta os primeiros resultados para a correlação canônica.

Tabela 8 - Correlação canônica e teste de significância

\begin{tabular}{|c|c|c|c|c|c|c|}
\hline $\begin{array}{c}\text { Função } \\
\text { Canônica }\end{array}$ & $\begin{array}{c}\text { Correlação } \\
\text { Canônica (R) }\end{array}$ & $\mathrm{R}^{2}$ & Qui-quadrado & $\mathrm{GL}$ & Sig. & $\lambda$ de Wilks \\
\hline 1 & 0,8371 & 0,7007 & 88,0653 & 20 & 0,0000 & 0,1779 \\
\hline 2 & 0,5696 & 0,3245 & 26,5410 & 12 & 0,0090 & 0,5943 \\
\hline 3 & 0,3295 & 0,1085 & 6,5373 & 6 & 0,3658 & 0,8797 \\
\hline 4 & 0,1149 & 0,0132 & 0,6778 & 2 & 0,7125 & 0,9868 \\
\hline
\end{tabular}

Fonte: Dados da pesquisa, 2010.

Para decidir qual função canônica analisar são necessários três critérios. Segundo Hair Jr. et al. (2007, p.367), "os três critérios são (1) nível de significância estatística da função, (2) magnitude da correlação canônica e (3) medida de redundância para o percentual de variância explicada a partir dos dois conjuntos de dados". Com base na Tabela 8, pode-se afirmar que a função 1 
apresenta índice de correlação canônica substancialmente maior que as outras, o que a leva a ter maior raiz canônica, além de significância estatística também maior, apesar de outra função ter apresentado significância estatística ao nível de 1\%. Com isso, a função 1 foi a escolhida para ser analisada neste estudo.

A correlação canônica mede a força da associação entre os dois conjuntos de variáveis. A força do relacionamento entre os pares de variáveis é refletida principalmente pelo índice de correlação canônica da função escolhida. O valor de 0,8371 indica forte correlação positiva e significativa. Quando elevado ao quadrado, tal coeficiente representa a quantidade de variância em um composto linear da função canônica atribuída a outro composto da mesma função. Sendo assim, pode-se dizer que, $70,07 \%$ da variação em um composto linear da primeira função são influenciados pela variação no outro composto que a compõe.

Ainda, faz-se necessário analisar o índice de redundância da função escolhida, que se apresenta a seguir:

Tabela 9 - Índices de redundância

\begin{tabular}{|l|r|r|r|}
\hline \multicolumn{1}{|c|}{ Variáveis } & $\begin{array}{c}\text { Variância } \\
\text { Compartilhada }\end{array}$ & $\mathrm{R}^{2}$ & Redundância \\
\hline Dependentes & 0,4424 & 0,7007 & 0,3100 \\
\hline Independentes & 0,4474 & 0,7007 & 0,3135 \\
\hline
\end{tabular}

Fonte: Dados da pesquisa, 2010.

Os índices de redundância apresentam valores representativos onde 31,00\% de variância nas variáveis dependentes é explicada pela variável estatística canônica independente e 31,35\% de variância nas variáveis independentes é explicada pela variável estatística canônica dependente.

Por fim, para identificar o tipo de relação entre os fatores de cultura e clima organizacionais, serão analisados os resultados das cargas e dos pesos canônicos.

Tabela 10 - Cargas e Pesos Canônicos

\begin{tabular}{|l|r|r|}
\hline Variáveis & Cargas Canônicas & Pesos Canônicos \\
\hline$Y_{1}$ Apoio da chefia e da organização & 0,9577 & 0,8060 \\
\hline$Y_{2}$ Recompensa & 0,8109 & 0,2723 \\
\hline$Y_{3}$ Conforto físico & 0,4402 & 0,0578 \\
\hline$Y_{4}$ Controle/Pressão & $-0,2431$ & $-0,2376$ \\
\hline$Y_{5}$ Coesão entre colegas & 0,6200 & 0,0403 \\
\hline & & \\
\hline Independentes & & 0,5301 \\
\hline$X_{1}$ Cultura Clan & 0,9027 & 0,5661 \\
\hline$X_{2}$ Cultura Inovativa & 0,8670 & $-0,3132$ \\
\hline$X_{3}$ Cultura de Mercado & $-0,2146$ & 0,2326 \\
\hline$X_{4}$ Cultura Hierárquica & 0,4209 & \\
\hline
\end{tabular}

Fonte: Dados da pesquisa, 2010.

Os coeficientes da equação acima são os "pesos canônicos" e são similares aos "betas" de uma regressão múltipla. Entretanto, o uso dos pesos para analisar uma função canônica pode acarretar em alguns problemas como instabilidade dos valores em função da amostra e dificuldades de interpretação dos valores em um ambiente com multicolinearidade. Isso nos leva 
a analisar a carga canônica para determinar a importância relativa das variáveis para cada composto canônico. Os altos valores das cargas canônicas, gerados para a maior parte das variáveis, reforça a viabilidade do modelo.

Com base na Tabela 10, verifica-se que os fatores culturais que mais influenciam positivamente os fatores de clima organizacional, para o caso estudado, são a cultura clã e a cultura inovativa. Isto talvez se deva ao fato de que a cultura clã incentiva o trabalho em grupo, os programas de envolvimento dos funcionários e o comprometimento corporativo com os empregados, além de enfatizar valores como coesão, moral, participação e abertura, criando uma relação quase paterna da organização com o funcionário, gerando segurança. Da mesma forma a cultura inovativa incentiva o trabalho dinâmico, empreendedor e criativo, encorajando a liberdade e iniciativa individual, promovendo um ambiente de trabalho onde os erros são tolerados. É importante destacar que essas duas tipologias culturais estão inseridas na dimensão flexibilidade, ou seja, direcionadas para a descentralização, o que pode ser um indicativo de que neste caso estudado os respondentes teriam uma melhor percepção do clima organizacional se tivessem maior autonomia em suas atividades.

Outro dado que pode ser extraído da Tabela 10 é que a cultura de mercado também tem uma relação bastante forte com a percepção de clima organizacional, porém é uma relação negativa, ou seja, no caso analisado, quanto maior a presença da cultura de mercado, menor o valor de clima atribuído pelos funcionários. Tem-se que a cultura de mercado é voltada para os resultados, onde alcançar as metas estabelecidas, melhorar a produtividade e gerar rendimentos são ações fundamentais. Isto pode gerar um desconforto para os indivíduos devido à grande pressão exercida pela organização, o que foi, inclusive, evidenciado pelo fato do fator de clima "Controle/Pressão" estar diretamente relacionado a esta tipologia cultural (seus valores de carga e peso canônicos estão negativos).

\section{CONCLUSÃO}

Com base nos resultados acima, pode-se afirmar que, para a organização analisada, houve forte influência da cultura organizacional sobre o clima organizacional, o que comprova empiricamente aquilo que é defendido por vários pesquisadores, como, por exemplo: Santos (1999), Tamayo (1999), Martins et al. (2004), Martins (2008), Schneider (1985), Souza (1978) e Puente-Palacios (2002). Outra evidência é que uma cultura "forte" pode gerar uma melhor percepção do clima organizacional.

Porém, algumas discussões podem ser realizadas:

a) Os resultados não confirmam a hipótese do modelo CVM sobre o equilíbrio entre as culturas e demonstram, para o caso analisado, que as tipologias culturais clã e inovativa deveriam ser enfatizadas em detrimento da cultura de mercado com o intuito de melhorar a percepção de clima da organização pelos indivíduos. Entretanto, Santos (1998) cita que a ênfase exagerada em aspectos específicos de algumas tipologias pode gerar "pontos cegos". O cuidado exagerado em desenvolver e fazer aflorar os traços da cultura clã pode gerar participação inapropriada, discussões improdutivas, individualismo descontrolado e excesso de permissividade. Já para a cultura inovativa 
os pontos negativos seriam os conflitos, hostilidades e oportunismo político. Os presentes resultados não permitem identificar esses "pontos cegos".

b) Os resultados apontam para a existência de subculturas dentro da organização analisada, uma vez que a análise de clusters dos dados relativos à cultura organizacional gerou uma divisão, formando um grupo com cultura "forte" e outro grupo com cultura "fraca". Este resultado coaduna à opinião de Schein (1992) e vai de encontro ao exposto por Martins (et al. 2004, 2008) quando a autora afirma que uma mesma organização pode possuir vários climas em diferentes setores, mas possui uma só cultura.

c) O presente estudo nos permite refutar a influência de aspectos pessoais como idade, sexo, estado civil, tempo de trabalho na organização, cargo, escolaridade e o fato de ocupar ou não função de chefia aos valores atribuídos pelos respondentes à cultura e ao clima organizacionais, o que pode ser um indicativo de que os elementos da cultura e do clima organizacional estão relacionados a percepções compartilhadas acerca da organização. Este fato pode, ainda, reafirmar a diferença entre clima psicológico e clima organizacional (JAMES; JONES, 1974, MARTINS et al., 2004, MARTINS, 2008).

Diante do exposto, considera-se que este estudo mostra-se útil no auxílio da gestão da cultura organizacional, pois, esta apresenta-se como problema pertinente nas organizações contemporâneas que precisam mudar ou reforçar sua cultura organizacional visando o alcance de vantagens competitivas. Da mesma maneira, colabora para o entendimento de como fatores culturais influenciam o clima das organizações, sendo este considerado outro gerador de vantagens competitivas nas organizações.

Tem-se ainda que, apesar da forte influência da cultura organizacional sobre o clima da organização constatada neste estudo, trata-se de construtos diferentes e que, em momento algum, corre-se o risco de ser redundante (PUENTE-PALACIOS, 2002, MARTINS et al., 2004, MARTINS, 2008). Porém, é importante frisar, que o meio acadêmico ainda carece de estudos que realizem uma investigação da validade discriminante entre as medidas desses dois construtos.

Por fim, vale a pena ressaltar que os resultados alcançados neste estudo somente podem ser generalizáveis para a organização em foco (um dos campus do Instituto Federal de Ensino de um estado do nordeste brasileiro) e que o fato dos construtos em análise serem mutáveis ao longo do tempo, pode requerer um estudo longitudinal para melhor descrever a dinâmica de suas relações.

\section{REFERÊNCIAS}

1. CAMERON, K. S.; QUINN, R. E. Diagnosing and changing organizational culture. San Francisco: Jossey-Bass, 2006.

2. CASTRO, M. L. P. Fatores significativos do clima organizacional, na percepção dos docentes de uma instituição de ensino superior privada. Dissertação de Mestrado - Universidade Federal de Santa Catarina. Florianópolis, 2003.

3. CODA, R. Pesquisa de clima organizacional e gestão estratégica de recursos humanos. In: BERGAMINI, C. W.; CODA, R. Psicodinâmica da vida organizacional: motivação e liderança. 
São Paulo: Atlas, 1997.

4. DEAL, T.; KENNEDY, A. Corporate Culture: the rites and rituals of corporate life. Massachusets: Addison-Wesley, 1982.

5. FOREHAND, G. A.; GILMER, B. H. Environmental variation in studies of organizational behavior. Psychological Bulletin, v. 62, n. 6, dec. 1964.

6. FREITAS, M. E. Cultura Organizacional: identidade, sedução e carisma? 4. ed. Rio de Janeiro: Editora FGV, 2005.

7. FREITAS, M. E. Cultura Organizacional: formação, tipologias e impacto. São Paulo: Makron Books, 1991.

8. HAIR JR., J. F.; ANDERSON, R. E.; TATHAN, R. L.; BLACK, W. C. Análise multivariada de dados. 5.ed. São Paulo: Bookman, 2007.

9. HOFSTEDE, G. Culturas e organizações - compreender a nossa programação mental. Lisboa: Sílabo, 1991.

10. JAMES, L. R.; JONES, A. P. Organizational climate: a review of theory and research. Psychological Bulletin, v. 81, n. 12, p. 1096-1112, 1974.

11. KATZ, D.; KAHN, R. Psicologia Social das Organizações. São Paulo: Atlas, 1985.

12. MALHOTRA, N. K. Pesquisa de marketing: uma orientação aplicada. 3.ed. Porto Alegre: Bookman, 2001.

13. MARTINS, M. C. F. et al. Construção e validação de uma escala de medida de clima organizacional. Revista Psicologia Organizações e Trabalho, Florianópolis, v. 4, n. 1, p.37-60, jan. 2004.

14. MARTINS, M. C. F. Clima Organizacional. In: SIQUEIRA, M. M. M. (Org.) Medidas do Comportamento Organizacional. Porto Alegre: Bookman, 2008.

15. MELLO, M. S. O. A qualidade do clima organizacional como variável interveniente no desempenho humano no trabalho: um estudo de caso da empresa Herbarium. Dissertação de Mestrado - Universidade Federal de Santa Catarina. Florianópolis, 2004.

16. OLIVEIRA, M. A. Pesquisas de clima interno nas empresas: o caso dos desconfiômetros avariados. São Paulo: Nobel, 1995.

17. PETERS, T.; WATERMAN JR., R. In search of excellence. New York: Harper and How, 1982.

18. PUENTE-PALACIOS, K. E. Abordagens teóricas e dimensões empíricas do conceito de clima organizacional. Revista de Administração, v. 37, n. 3, p. 96 - 104, jul./set. 2002.

19. RIZZATTI, G. Categorias de análise de clima organizacional em universidades federais brasileiras. Tese de doutorado - UFSC. Florianópolis, 2002.

20. SÁ leitÃO, J. S.; GUIMARÃES, T. A.; ROSAL, M. A. A. Metodologia de diagnóstico de clima organizacional em ambiente de inovação tecnológica. In: Encontro da Associação Nacional de Pós-Graduação em Administração. 22, 1998, Foz do Iguaçu. Anais eletrônicos. Disponível em: <http://www.anpad.org.br>. Acesso em 11/05/2009.

21. SANTOS, N. M. B. F. Cultura organizacional e desempenho: pesquisa, teoria e aplicação. Lorena: Stiliano, 2000.

22. SANTOS, N. M. B. F. Clima organizacional: pesquisa e diagnóstico. Lorena: Stiliano, 1999. 
23. SANTOS, N. M. B. F. Cultura e desempenho organizacional: um estudo empírico em empresas brasileiras do setor têxtil. Revista de Administração Contemporânea. São Paulo, v. 2, n. 1, p. 47-66, jan./abr. 1998.

24. SANTOS, N. M. B. F. Impacto da cultura organizacional no desempenho das empresas, conforme mensurado por indicadores contábeis: um estudo interdisciplinar. Tese de doutorado - FEA/USP. São Paulo, 1994.

25. SCHEIN, E. H. Organizational culture and leadership. California: Jossey-Bass, 1992.

26. SCHEIN, E. H. Coming to a new awareness of organizational culture. Sloan Management Review, v.25, p. 3-16, 1984.

27. SCHNEIDER, B. Organizational Behavior. Annual Review of Psychology, v. 36, p. 573-611, jan. 1985.

28. SMIRCICH, L. Concepts of culture and organizational analysis. Administrative Science Quaterly, v. 28, p.339-358, 1983.

29. SOUZA, E. L. P. Clima e cultura organizacionais: como se manifestam e como se manejam. São Paulo: Edgard Blucher, 1978.

30. TAMAYO, A. Valores e clima organizacional. In: PAZ, M. G. T.; TAMAYO, A. (Orgs). Escola, saúde mental e trabalho. Brasília: Editora UnB, 1999.

31. VERBEKE, W.; VOLGERING, M.; HESSELS, M. Exploring the conceptual expansions within the field of organizational behaviour: organizational climate and organizational culture. Journal of Management Studies, v. 35, n. 3, p. 303-329, 1998.

32. VERGARA, S. C. Projetos e relatórios de pesquisa em administração. 3. ed. São Paulo: Atlas, 2000. 